Revue internationale P.M.E.

Économie et gestion de la petite et moyenne entreprise

Revure

internationale

PME

\title{
Les politiques de formation de PME françaises de classe mondiale
}

\section{Agnès Paradas et Olivier Torrès}

Volume 9, numéro 2, 1996

URI : https://id.erudit.org/iderudit/1008260ar

DOI : https://doi.org/10.7202/1008260ar

Aller au sommaire du numéro

Éditeur(s)

Presses de l’Université du Québec

ISSN

0776-5436 (imprimé)

1918-9699 (numérique)

Découvrir la revue

Citer cet article

Paradas, A. \& Torrès, O. (1996). Les politiques de formation de PME françaises de classe mondiale. Revue internationale P.M.E., 9(2), 7-35.

https://doi.org/10.7202/1008260ar

\section{Résumé de l'article}

Cette étude présente une recherche empirique sur la formation professionnelle dans des PME françaises classées au premier rang pour la part de marché mondial qu'elles détiennent dans leur domaine d'activité. Dans un contexte d'intégration croissante des économies, bon nombre de PME tirent profit de ce processus de globalisation et s'accaparent parfois le leadership d'une activité à l'échelle mondiale. Ces PME présentent ainsi des caractéristiques communes. Les auteurs tentent de faire ressortir les relations pouvant exister entre les modalités de la formation suivie et les éléments de stratégie des PME décrites. Les résultats permettent ainsi de percevoir que les PME mondiales ont le plus souvent adopté des politiques de formation à caractère d'investissement, ce qui n’est pas le cas de la majorité des PME françaises. La compréhension des raisons de ces engagements donne tout son intérêt à la présente recherche et permet de dégager des éléments qui président à l'existence de telles formations. Il apparaît ainsi que l'innovation permanente, la spécialisation et la technologie déterminent fortement les politiques de formation existantes.
Tous droits réservés @ Presses de l’Université du Québec, 1996
Ce document est protégé par la loi sur le droit d'auteur. L'utilisation des services d’Érudit (y compris la reproduction) est assujettie à sa politique d'utilisation que vous pouvez consulter en ligne.

https://apropos.erudit.org/fr/usagers/politique-dutilisation/ 


\title{
Les politiques de formation de PME françaises de classe mondiale
}

\author{
Agnès PARADAS \\ Institut universitaire technologique (IUT) de Valence \\ Olivier TORRÈS \\ Université Paul-Valéry de Montpellier
}

MOTS CLÉS

\section{Formation - PME - Mondialisation - Stratégie de globalisation Haute technologie - Spécialisation}

\begin{abstract}
RÉSUMÉ
Cette étude présente une recherche empirique sur la formation professionnelle dans des PME françaises classées au premier rang pour la part de marché mondial qu'elles détiennent dans leur domaine d'activité. Dans un contexte d'intégration croissante des économies, bon nombre de PME tirent profit de ce processus de globalisation et s'accaparent parfois le leadership d'une activité à l'échelle mondiale. Ces PME présentent ainsi des caractéristiques communes. Les auteurs tentent de faire ressortir les relations pouvant exister entre les modalités de la formation suivie et les éléments de stratégie des PME décrites. Les résultats permettent ainsi de percevoir que les PME mondiales

\section{LES AUTEURS}

Agnès Paradas, maître de conférences à l'Institut universitaire technologique (IUT) de Valence, lequel dépend de l'Université Pierre-Mendès-France de Grenoble, docteure en sciences de gestion, enseigne le marketing et intervient en GRH. Elle est également membre de l'ERFI (Équipe de recherche sur la firme et l'industrie) à Montpellier I, où elle mène des travaux sur la GRH en PME et TPE et, plus particulièrement sur la formation professionnelle. Olivier Torrès, ancien élève de l'École normale supérieure de Cachan, professeur agrégé (PRAG) en économie et gestion à l'Université Paul-Valéry de Montpellier, enseigne essentiellement le management de la firme internationale. Il est membre de l'ERFI depuis 1993 au sein duquel il effectue des travaux sur le thème des stratégies de globalisation des PME. Adresse: ERFI, Faculté de sciences économiques, 14, rue du Cardinal-de-Cabrières, 34 060, Montpellier Cedex, France.

Les auteurs tiennent à remercier les lecteurs anonymes pour la justesse de leurs recommandations et les multiples remarques qui ont été intégrées tout au long de leur travail.
\end{abstract}


ont le plus souvent adopté des politiques de formation à caractère d'investissement, ce qui n'est pas le cas de la majorité des PME françaises. La compréhension des raisons de ces engagements donne tout son intérêt à la présente recherche et permet de dégager des éléments qui président à l'existence de telles formations. Il apparaît ainsi que l'innovation permanente, la spécialisation et la technologie déterminent fortement les politiques de formation existantes.

\begin{abstract}
This study presents an empirical research on professional training in French small businesses with a worldwide dimension, leaders in their activity. Small businesses can take advantage of the actual economic integration process and can assume the leadership of one activity on an international scale. So, these small businesses have common characteristics. We try to put forward the relations between training policy and some strategic small businesses data. We show that French small businesses with worldwide dimension are often making training investments, contrary to the majority of small businesses. The understanding of this attitude can give determinant material to such training policy. Thus, continuous innovation, specialization and technology may explain training policy.
\end{abstract}

\title{
RESUMEN
}

Esta comunicacion presenta un estudio empirico sobre la formacion en las pequeñas empresas francesas que son liberes mundiales en su sector. Las pequeñas empresas pueden sacar ventaja del proceso del globalizacion y tomar el puesto de leader dentro de una actividad que se desarolla a escala mundial. Poseen determinadas caracteristicas en comùn.

Intentamos mostrar las relaciones que existen entre politicas de formacion y elementos de la estrategia de las pequeñas empresas. Mostramos que, al contrario de la mayoria de las pequeñas empresas, las que tienen una actividad mundial de leader realizan mas a menudo inversiones de formacion. El estudio de esta caracteristica puede dar mayores explicaciones sobre politica de formacion como elemento de inversion. De esta forma parece ser la inovacion permanente, especialization y la tecnologia pueden explicar tales politicas de formacion.

\section{Introduction}

La formation professionnelle dans les PME françaises évolue lentement depuis la loi de 1971 imposant une contribution au titre de la formation continue. Nombre d'entre elles suivent encore des politiques de formation très ponctuelles n'entrant pas dans une logique d'investissement en formation (CEREQ, 1992 ; AGEFOS, 1992). Bel et Rosanvallon (1991) proposent une typologie de ces politiques pour les PME, en distinguant les PME ne faisant aucune 
formation, soit celles «épuisant la taxe», des PME dites «innovantes » pour lesquelles l'engagement financier est considéré comme une ressource mobilisable par l'entreprise pour accompagner ses choix stratégiques. Les modalités des politiques de formation reflètent les différentes manières de conduire les actions de formation dans l'entreprise. De façon caricaturale, il est possible d'opposer deux profils types extrêmes. D'une part apparaissent les politiques de formation ponctuelles mises en place sans réelle réflexion sur les actions engagées et les résultats escomptés ; une logique réactive, curative et adaptative guide ces actions. La formation reste peu considérée et le pourcentage obligatoire versé au titre de la formation continue semble davantage perçu comme une dépense supplémentaire que comme une occasion d'amélioration. D'autre part, il existe des politiques de formation menées dans une perspective d'investissement. Les notions d'investissement stratégique (Mathe et Rivet, 1988; Paradas, 1993) et d'investissement en formation (Caspar et Afriat, 1988; Walther, 1988) ne revêtent pas le même sens pour tous. Toutefois, il reste possible de considérer globalement que la politique de formation est menée dans une logique d'investissement dans les cas où la réflexion se fait à long terme, quand celle-ci s'intègre dans une stratégie de l'entreprise, tout en tenant compte des besoins des salariés. Cette perspective se situe alors davantage dans une logique proactive, réfléchie et délibérée.

Or, peu d'éclairages existent sur les origines de cette diversité, même si les travaux de certains auteurs (Bel et Rosanvallon, 1990; Paradas, 1993) laissent penser que d'autres variables en plus de la taille caractérisent des politiques de formation différentes dans les PME. Par conséquent, l'étude d'une population particulière de PME doit permettre de relever certaines caractéristiques distinctives déterminantes des politiques de formation. En effet, « les besoins particuliers en matière de formation continue dépendent intimement des stratégies de l'entreprise, et de l'organisation et de la gestion du travail qui lui sont propres» (Caron, 1994).

En 1986, sous l'impulsion du ministre du Commerce extérieur français, une association destinée à promouvoir le commerce extérieur français voit le jour: «le club des numéros 1 mondiaux». Les responsables régionaux du ministère sélectionnent les entreprises à partir d'un critère simple : la détention de la plus grande part du marché mondial. À ce jour, deux cents entreprises ont accepté d'adhérer à ce club d'élite. Parmi elles, un cinquième présente un effectif inférieur à deux cents salariés. La plus petite d'entre elles est composée de seulement six salariés, bien qu'elle soit leader mondial dans la fabrication sur mesure de fibres optiques à infrarouge. Cet exemple démontre combien il serait erroné de conclure que la mondialisation des marchés se réduit à la seule problématique des économies d'échelle et de la standardisation des produits (Levitt, 1983). Dans le présent article, nous nous 
proposons d'étudier les politiques de formation de ces PME de classe mondiale.

\section{Cadre de l'étude}

\subsection{Problématique}

\subsubsection{Des PME spécifiques}

Les PME internationales ne constituent pas forcément un ensemble homogène (Cavusgil, 1984 ; Joyal et Ramangalahy, 1993). Il convient donc de repérer les variables clés qui caractérisent l'ensemble des PME du club des numéros 1 mondiaux.

Ces PME se caractérisent toutes par un fort engagement à l'exportation puisqu'elles occupent le premier rang en termes de part du marché mondial. Une très forte proportion ( $93 \%$ ) de ces entreprises exporte dans plus de cinq pays différents, parfois même dans le monde entier, couvrant jusqu'à plus de 90 pays à la fois, et $85 \%$ d'entre elles possèdent un service d'exportation. L'existence d'un service export constitue un indice significatif du degré d'engagement à l'international (Monnoyer-Longé, 1990 ; Bayad, 1992). En ce sens, les PME du club des numéros 1 mondiaux sont fortement engagées dans le processus d'internationalisation. Elles sont à la pointe de l'engagement croissant des PME dans le commerce international évoqué par de nombreuses études (Bucaille et Costa de Beauregard, 1987; Malivernay, 1991; Wagner, 1995). Ces performances internationales sont d'autant plus compréhensibles que $80 \%$ des dirigeants considèrent que l'étroitesse du marché national ne leur permettrait pas d'atteindre le seuil de rentabilité. La saturation du marché intérieur peut rapidement amener l'entreprise à dépasser les frontières nationales (Saporta, 1986; Porter, 1993). Prenant à contre-pied les conceptions séquentielles du processus d'exportation des PME, la très grande majorité de ces PME de classe mondiale ont envisagé l'exportation très tôt, certaines d'entre elles $(45 \%)$ ayant exporté l'année même de leur création.

Toutefois, il serait excessif de réduire l'internationalisation de l'entreprise au seul aspect de l'exportation, même si cette tendance est courante dans le champ des PME (Fourcade, 1993). Une étude récente montre que le processus d'internationalisation des PME de l'industrie italienne du vêtement concerne tout autant les activités commerciales que celles de production (Berra et al., 1995). Ces remarques attirent notre attention sur d'autres aspects de l'internationalisation de la PME. Ainsi, l'entreprise importatrice peut être qualifiée d'internationale au même titre que celle qui exporte (Saporta, 1993) et $60 \%$ des entreprises interrogées importent une partie de leurs approvisionnements parfois dans des proportions importantes (taux d'importation 
dépassant $90 \%$ des fournitures totales). Cette forte orientation internationale des activités marchandes conduit la plupart des dirigeants de ces entreprises à se déplacer fréquemment à l'étranger, passant en moyenne deux mois à sillonner la planète pour des motifs de prospection ou d'innovation. À l'instar de globe-trotters, $50 \%$ d'entre eux déclarent passer entre un mois et deux mois à l'étranger et $25 \%$ entre trois et six mois. L'espace de travail des dirigeants semble également fortement internationalisé.

La forte mobilité internationale des dirigeants, la précocité et l'importance des opérations d'importation et d'exportation, l'insuffisance du marché national, l'intensité de la concurrence étrangère confèrent à ces entreprises un espace économique naturellement mondialisé. Les stratégies internationales de ces PME ont une portée plus large que les simples stratégies d'exportation. Ces PME s'apparentent à des entreprises globales (Torrès, 1994). En effet, « une entreprise se transnationalise tout autant pour des raisons d'élargissement des marchés que pour des raisons d'optimisation des conditions de production et de gestion » (Fuguet et al., 1986). La compétitivité de ces entreprises est recherchée d'emblée à l'échelle mondiale. En définissant la stratégie de globalisation comme le stade ultime de l'internationalisation où l'espace de travail de l'entreprise se confond avec le marché internationalisé et transnational (Joffre, 1994), nous pouvons considérer que les PME du club des numéros 1 mondiaux adoptent de véritables stratégies de globalisation puisqu'elles se caractérisent non seulement par un espace de marché mondial, mais également par un espace de fonctionnement ${ }^{1}$ qui dépasse les frontières nationales (figure 1 ).

\subsubsection{Articulation de la problématique autour du métier et de la mission}

Plusieurs auteurs considèrent que l'internationalisation peut constituer un choc qui implique de profonds changements dans le mode de fonctionnement de la PME (Walters et Samiee, 1990 ; Moini, 1992 ; Guilhon et al., 1993). L'internationalisation de la PME n'est pas sans avoir d'effet sur les pratiques de gestion des ressources humaines (Hermel, 1991 ; Bayad, 1992) et elle suscite généralement des pratiques de formation appropriées (Sarnin, 1990). D'autant plus que le problème de la disponibilité et de la formation des employés peut être considéré comme «l'un des premiers freins au développement international » (Joffre, 1987). Lorsque le champ d'activité devient mondial, la maîtrise des

1. L'espace de fonctionnement est défini par la localisation de l'ensemble des intrants de l'entreprise et de ses fournisseurs, c'est-à-dire l'ensemble des ressources humaines, financières, matérielles et immatérielles qui interviennent dans le processus de production (Fuguet et al., 1986). 
FIGURE 1

Stratégies d'exportation et de globalisation

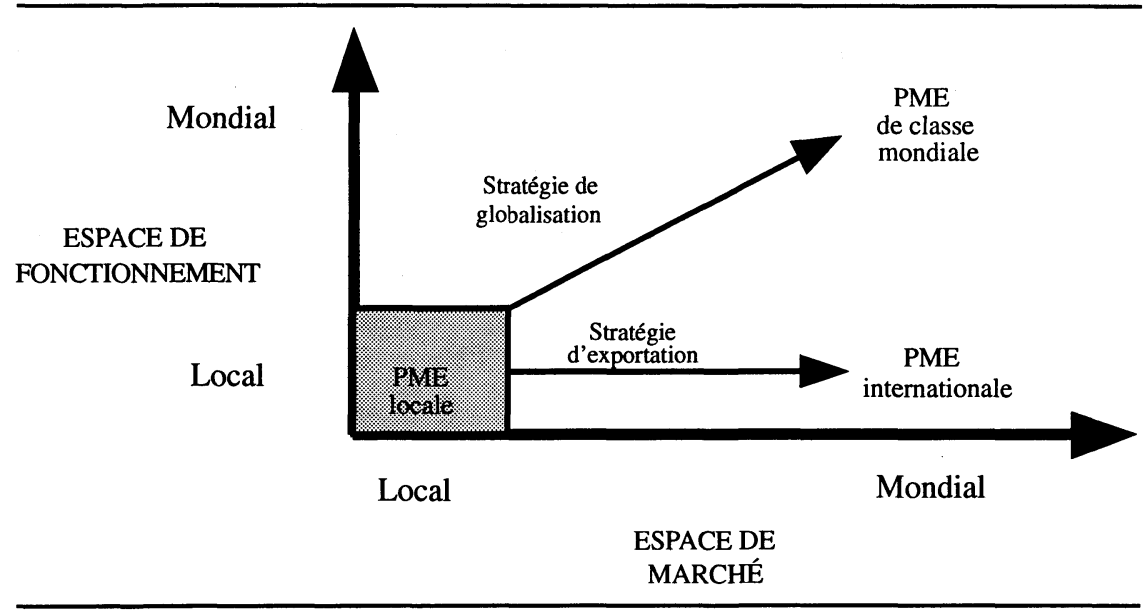

Source: Torrès, 1994.

opérations exige de nouveaux efforts et sollicite des compétences entrepreneuriales plus importantes. La formation permet alors d'élargir les compétences de l'entreprise pour maîtriser ces changements (Guilhon et al., 1993). Partant de ce principe et puisque les stratégies de globalisation traduisent une extension de la dimension internationale au sein de l'entreprise, il est probable que ce type de stratégie renforce l'ampleur et la nature du changement et suscite encore plus ce besoin de formation. Dans ces conditions, il est possible de déduire que les PME admises au club des numéros 1 mondiaux investissent activement dans la formation des ressources humaines. Elles représentent, par conséquent, des lieux privilégiés pour analyser la formation.

De plus, les PME du club des numéros 1 mondiaux ressemblent aux « courtiers-stratèges » décrits par Reich (1993), dont la base de compétitivité est de «trouver le bon accord entre une technologie spécifique et des besoins spécifiques », ce qui leur permet de garder une position confortable sur le marché. Les modes de fonctionnement et d'organisation des PME étudiées s'effectuant à une échelle mondiale, ils exigent la maîtrise d'un métier (un savoir-faire) très spécifique qui évolue en fonction des changements technologiques constants. Parallèlement, la mission (le savoir-satisfaire) de ces PME repose sur la découverte d'un besoin particulier dans une sphère élargie et nécessite une veille perpétuelle et active, pour bien percevoir les évolutions permanentes des marchés. La stratégie de globalisation consiste alors «à appréhender de façon globale un système de métiers et un système de marchés géographiques [...]. 
La globalisation n'a de sens que si elle est anticipative et prospective, c'est-àdire porteuse d'une vision de l'avenir abordant l'évolution future d'une industrie, d'un métier et d'un marché. » (Deroy et Lagrand-Escure, 1992) Ainsi, il apparaît que les stratégies de globalisation qui caractérisent ces entreprises suscitent une maîtrise accrue, voire une modification sensible, du couple métier-mission.

Or, différents travaux sur la formation montrent précisément que les évolutions des couples métier-mission, soit à travers des changements technologiques et organisationnels, soit par des mutations dans le positionnement concurrentiel, ont une influence sur la configuration des politiques de formation (Castagnos, 1990 ; Tache et Dejean, 1991 ; Paradas, 1993). Plus particulièrement, selon Beccarelli et Perker (1991) «les variables qui jouent positivement en faveur de l'investissement en formation seraient la politique commerciale de l'entreprise vis-à-vis des marchés internes et de l'export ainsi que la priorité donnée aux nouveaux produits et donc à la recherche». Il semblerait que l'existence conjointe d'une évolution des métiers vers une plus grande complexité technique et organisationnelle et d'une évolution de la mission à travers les changements dans les produits ou les marchés soit à l'origine d'une politique de formation plus intégrée à la stratégie générale et de long terme, c'est-à-dire se rapprochant d'une logique d'investissement. La globalisation apparaît comme un contexte particulièrement propice à l'élaboration de politique de formation à caractère d'investissement. Une relation entre le profil des PME de l'échantillon étudié et les politiques de formation suivies émerge clairement.

Par conséquent, la présente étude envisage de montrer dans quelle mesure les PME de classe mondiale élaborent plutôt des politiques de formation à caractère d'investissement que des politiques de formation ponctuelles. Il s'agira alors, si les observations justifient cette proposition, de s'interroger sur les caractéristiques de l'échantillon ayant une influence sur ces orientations. Ce qui nous amènera à approfondir la question suivante : quelles sont les caractéristiques des PME de classe mondiale qui déterminent les politiques de formation suivies?

À l'instar des travaux de Rousseau et de Wade Benzoni (1994), lesquels relient la stratégie des entreprises aux pratiques de GRH, l'objet de cette étude est d'analyser la relation entre la stratégie de globalisation des PME de classe mondiale et les pratiques de formation. La mise en évidence de facteurs explicatifs des politiques de formation à caractère d'investissement contribuerait à renforcer la thèse d'une nécessaire approche contingentielle de la GRH, en général (Fabi et Garand, 1994), et des pratiques de formation, en particulier (Meschi, 1993). 


\section{2. Éléments théoriques et méthodologiques}

\subsection{1. Étendue et modalités de formation}

La dépense, en pourcentage de la masse salariale destinée à la formation, constitue un indicateur significatif de l'étendue de la formation. En France, l'obligation légale s'élève à $1,5 \%$ de la masse salariale dans les entreprises de plus de 10 salariés et à $0,15 \%$ pour des effectifs moindres. Cependant, il arrive que certaines entreprises choisissent de consacrer à la formation un pourcentage supérieur.

L'utilisation de plusieurs autres variables permet de déterminer plus précisément l'existence d'une logique d'investissement en formation. L'existence d'un plan de formation, son caractère flexible ou rigide et son horizon temporel constituent de précieux indicateurs de l'attitude de la PME en matière de formation. Toutes les PME n'ont pas un plan de formation. La planification, de manière générale, est d'ailleurs assez rare (d'Amboise et Bakanibona, 1990 ; d'Amboise, 1994). Pourtant, il semblerait qu'une formalisation, même réduite par rapport aux grandes entreprises, ainsi qu'une réflexion dans la durée soient significatives d'une action de formation plus développée (Bel et Rosanvallon, 1991). De plus, pour Verdier (1991), l'articulation des actions formelles et informelles de formation dans la PME constitue une amélioration de la situation de formation.

Il s'agira également d'étudier les contenus des formations suivies et leur nature. Ce sont les variétés des contenus de formation et leur relation avec l'activité qui permettent de mieux apprécier les profils de formation. En ce qui concerne la nature des formations (stages internes, externes, de courte ou de longue durée, etc.), Bel et Rosanvallon (1990) remarquent que les PME innovantes ont davantage recours à l'externalisation. De plus, il semblerait qu'elles combinent les différentes formes. Enfin, d'après les résultats de l'AGEFOS (1992a), la taille semble déterminante du choix «interne/ externe».

Il est nécessaire de connaître la répartition de la responsabilité de la formation dans les PME, laquelle se fait principalement entre le chef d'entreprise, les responsables internes ou ceux des Fonds d'assurance formation (FAF). Les entreprises considérant le plus la formation comme un investissement ont davantage de responsables de ressources humaines ou de formation (entreprises du type A, selon Tache et Dejean, 1991). Les FAF jouent également un rôle important dans la gestion de la formation et peuvent assister la PME en tant que médiateur ou conseil (Dejean et Willems, 1991).

L'égalité d'accès à la formation pour les différents niveaux hiérarchiques et les différentes fonctions a un contenu informatif certain : la multiplication 
des acteurs est significative de la considération accrue de la formation dans les PME que Bel et Rosanvallon (1990) citent comme innovantes. Souvent, dans la PME, l'accès à la formation est très inégal, selon les individus de l'entreprise (Berton et Podevin, 1991). L'étude doit alors s'attacher à relever les différentes catégories faisant l'objet d'une action de formation : administratifs, productifs, cadres, employés, etc.

Enfin, une approche des obstacles perçus est indispensable pour une meilleure compréhension de la politique de formation suivie par les PME étudiées. Le plus souvent, de nombreux obstacles propres aux PME sont cités par les responsables interrogés (Bel et Rosanvallon, 1990; AGEFOS, 1992a ; Paradas, 1993). Les orientations de chaque réponse, significatives de l'existence de problèmes particuliers, viennent compléter les conclusions relatives aux politiques de formation engagées.

\subsubsection{Justification de la logique d'investissement et variables exogènes}

Différents énoncés de recherche ont été construits dans le but de relever des relations de cause à effet entre les caractéristiques des PME et leur politique de formation. Les responsables ont été interrogés sur les raisons de mise en place des formations dans leur PME. Ils ont eu le choix entre les propositions suivantes :

- l'évolution du niveau de technologie de l'entreprise, qui peut impliquer «des besoins en qualification rénovée» (Castagnos, 1990);

- la recherche de polyvalence pour une plus grande flexibilité (Paradas, 1993);

- l'ouverture internationale, dont les effets et les impacts sont possibles sur toutes les fonctions y compris la GRH et le management (Hermel, 1991 ; Bayad, 1992);

- l'adaptation aux clients et la normalisation (normes ISO, par exemple);

- la volonté de rester en position de leader, de maintenir son avantage concurrentiel (Porter, 1993);

- l'innovation permanente.

Si ces propositions ont été choisies de manière à être adaptées à l'échantillon retenu, il en est d'autres qui seraient applicables à toutes les PME. Ces dernières décrivent les caractéristiques des petites structures qui peuvent également influencer la politique de formation. Parmi ces caractéristiques, nous retiendrons comme variables exogènes: 
1. Le profil du dirigeant suivant son niveau de formation, son âge, son expérience: plusieurs auteurs notent l'importance de cette variable dans la détermination des modalités des politiques de formation. D'après Bel et Rosanvallon (1990), par exemple, la nature des politiques engagées est fortement liée à la personnalité du dirigeant. Son niveau de formation est très déterminant. Fourcade (1986) note que «paradoxalement, le besoin de formation est d'autant plus ressenti que le dirigeant est formé ou possède un niveau élevé de qualification». Ainsi, pour les PME mondiales, on peut supposer que si les dirigeants possèdent un haut niveau de formation, cela aura une influence sur leurs besoins et, par conséquent, sur leur politique de formation, sans pour autant qu'il y ait un lien avec la nature mondiale de leur marché. "Il est bien évident que l'orientation stratégique d'une PME est influencée par un ensemble de facteurs [...]. Selon Bamberger (dans d'Amboise et Fortin, 1990), on peut supposer que le choix d'une stratégie de compétition donnée est influencée par les caractéristiques personnelles du dirigeant, par sa personnalité, ses valeurs et ses attitudes. »

2. L'activité en termes d'industrie ou de service: il semblerait, par exemple, que les PME industrielles ont plus souvent un plan de formation que les autres (AGEFOS, 1992a).

3. La taille et le degré de maturité de l'entreprise: «plus la taille de l'entreprise et son degré de maturité sont élevés, plus les pratiques de gestion du personnel ont une dimension stratégique associée, entre autres, à la gestion prévisionnelle du personnel » (Bayad, 1992).

\subsubsection{Détermination de l'échantillon}

L'échantillon a été constitué à partir d'une population de 200 entreprises, toutes adhérentes en 1990, au «club des numéros 1 mondiaux ». Comme nous l'avons déjà vu, ce club, constitué à l'initiative du ministère français du Commerce extérieur, réunit diverses entreprises françaises à activité mondiale ayant choisi d'y adhérer. Une telle initiative tend à améliorer les conditions des entreprises en termes d'échange et de prestige, et, par ricochet, stimule en France le commerce international. Un cinquième de cette population, soit 40 entreprises, est représenté par des PME de moins de 200 salariés et peut être considéré comme la population mère. Parmi ces quarante PME, 29 ont répondu au questionnaire de la présente étude. Leur répartition par effectif et chiffre d'affaires en millions de francs peut se décliner comme indiqué au tableau 1. 
TABLEAU 1

Caractéristiques des PME étudiées

\begin{tabular}{lccc}
\hline Effectif & Nombre de PME & $\begin{array}{c}\text { Chiffre d'affaires } \\
\text { (en millions de francs) }\end{array}$ & Nombre de PME \\
\hline Moins de 10 & 2 & Moins de 10 & 2 \\
$11-50$ & 11 & $11-50$ & 14 \\
$51-100$ & 10 & $51-100$ & 7 \\
$100-200$ & 6 & $101-200$ & 4 \\
& & Sans réponse & 2 \\
\hline
\end{tabular}

Près de la moitié des entreprises étudiées sont des petites entreprises de moins de cinquante salariés. Signalons l'existence de deux toutes petites entreprises de moins de 10 salariés.

\section{Résultats descriptifs et comparatifs des modalités de formation}

Au-delà d'une simple description des pratiques des PME adhérentes au club des numéros 1 mondiaux, il peut s'avérer utile de rapprocher les résultats de l'étude à ceux d'autres enquêtes concernant les politiques de formation en PME (CEREQ, 1992 ; Paradas, 1993 ; AGEFOS, 1992a ; Bouayed, 1991 ; Bel et Rosanvallon, 1990 ; Bayad, 1992). Toutefois, l'hétérogénéité des échantillons et l'insuffisance des informations relatives aux dispositifs de recherche ne permettent pas d'établir de différences significatives à l'aide de tests statistiques. Par conséquent, ces rapprochements viseront essentiellement à indiquer des pistes de recherche.

\subsection{Des $P M E$ engagées dans la formation}

Toutes les PME françaises de classe mondiale interrogées déclarent faire de la formation. La comparaison avec la population générale des PME françaises indique une propension supérieure de ces entreprises mondiales à former. De Vendeuil (1992) affirme ainsi que 50\% des entreprises dont l'effectif se situe entre 20 et 50 salariés versent leur pourcentage sans rien entreprendre, et les travaux de l'AGEFOS montrent que les entreprises dont l'effectif se situe entre 10 et 500 salariés forment seulement en interne dans $52 \%$ des cas et ne forment pas du tout dans $9 \%$ des cas, ce qui correspond d'assez près aux tendances relevées par Fabi et Garand (1994, p. 335). 
TABleau 2

Montant des dépenses en formation

\begin{tabular}{lccccc}
\hline $\begin{array}{l}\text { Pourcentage } \\
\text { de la masse salariale } \\
\text { versé au titre } \\
\text { de la formation } \\
\text { continue }\end{array}$ & & Classe selon l'effectif & \multicolumn{1}{c}{$\begin{array}{c}\text { Total } \\
(\%)\end{array}$} \\
\hline & $1-10$ & $11-50$ & $51-100$ & $101-200$ & \\
\hline - moins de 1\% & 2 & 0 & 0 & 0 & $2(07,0 \%)$ \\
- entre 1\% et 2\% & 0 & 8 & 7 & 2 & $17(58,5 \%)$ \\
- entre 2\% et 5\% & 0 & 3 & 3 & 2 & $8(27,5 \%)$ \\
- plus de 5\% & 0 & 0 & 0 & 2 & $2(07,0 \%)$ \\
\hline
\end{tabular}

En ce qui concerne les dépenses engagées, les entreprises de l'échantillon dont l'effectif est compris entre 10 et 50 salariés se comportent à peu près de la même façon que celles observées par le CEREQ (1992) : elles sont presque $30 \%$ à dépenser plus que le pourcentage prescrit pour la formation. Cette allocation augmente proportionnellement selon la taille de l'entreprise : $30 \%$ des $51 / 100$ et $65 \%$ des $101 / 200$ dépensent entre 2 et $5 \%$ de leur masse salariale et parfois davantage pour la formation. Au total, la majorité des PME de classe mondiale se situe dans la norme habituelle en matière de dépenses obligatoires, mais une proportion non négligeable (35\%) dépasse les seuils fixés par la législation.

Nous retiendrons globalement de ces résultats un engagement actif en formation professionnelle. Au-delà de ces observations, il paraît intéressant de cerner le profil des politiques de formation engagées.

\subsection{Modalités de mise en cuvre des politiques de formation}

\subsubsection{Une formation centralisée et des contenus relativement communs}

En ce qui concerne les acteurs responsables de la formation, l'AGEFOS remarque qu'en PME, «la GRH relève de la direction », et que dans $69 \%$ des cas, ce sont les dirigeants de l'entreprise qui s'occupent directement de la formation. Malgré une propension à s'absenter de leur entreprise, $62 \%$ des responsables des PME mondiales affirment superviser les actions de formation, ce qui signifie que la formation conserve toute son importance. De plus, Tache et Dejean (1991) remarquent une forte présence des responsables de ressources humaines dans les PME qui investissent le plus en formation, sans préciser le sens de la causalité entre ces deux variables. Cela confirme la 
cohérence des résultats obtenus. Il semble ainsi que le caractère mondial des entreprises étudiées n'exerce pas d'influence sur les domaines de formation choisis, ni sur les acteurs concernés.

TABLEAU 3

Domaines de formation

\begin{tabular}{lr}
\hline $\begin{array}{l}\text { Domaines de formation } \\
\text { (plusieurs réponses possibles) }\end{array}$ & $\begin{array}{c}\text { Nombre } \\
\text { de réponses } \\
(\%)\end{array}$ \\
\hline - technique & $21(72 \%)$ \\
- informatique & $16(55 \%)$ \\
- langues & $15(51 \%)$ \\
- gestion & $8(27 \%)$ \\
- qualité & $7(24 \%)$ \\
\hline
\end{tabular}

L'analyse des contenus des formations fait ressortir les tendances suivantes : $72 \%$ des entreprises recourent à des formations dans des domaines techniques et plus de $50 \%$ dans des secteurs de l'informatique et des langues. Les autres formations citées sont plus rares. D'après Verdier (1991), «l'ordre de priorité des secteurs de formation où les entreprises entendent investir reste depuis les premiers pas du baromètre : l'informatique, les techniques, les langues ». Bouayed (1991) confirme cette idée dans le domaine des formations aux langues étrangères.

\subsubsection{Une formalisation poussée}

L'existence d'un plan de formation n'apparaît pas comme une évidence dans les PME (Fabi et Garand, 1994, p. 332). Dans les études de Bouayed (1991) et du CEREQ (1992), les PME qui élaborent un plan de formation représentent respectivement $40 \%$ et $34 \%$ des échantillons observés tandis que dans la présente étude, la proportion est de $65,5 \%$. Comme le montre le tableau cidessous, les PME mondiales ont tendance à formaliser assez souvent leurs actions de formation.

TABLEau 4

Existence et horizon temporel du plan de formation

\begin{tabular}{cccc}
\hline $\begin{array}{c}\text { Existence d'un } \\
\text { plan de } \\
\text { formation }\end{array}$ & $\begin{array}{c}\text { Nombre } \\
\text { d'entreprises } \\
(\mathbf{\%})\end{array}$ & $\begin{array}{c}\text { Horizon temporel } \\
\text { du plan de } \\
\text { formation }\end{array}$ & $\begin{array}{c}\text { Nombre } \\
\text { d'entreprises } \\
(\boldsymbol{\%})\end{array}$ \\
\hline non & $10(34,5 \%)$ & annuel & $11(58 \%)$ \\
oui & $19(65,5 \%)$ & pluriannuel & $8(42 \%)$ \\
\hline
\end{tabular}


De plus, l'horizon temporel de ces plans est plus étendu dans l'échantillon étudié ( $42 \%$ des plans sont pluriannuels) que dans l'échantillon observé par l'AGEFOS (seulement $33 \%$ ). Cela indique tout d'abord une plus grande capacité et une volonté de formaliser les actions de formation, de faire un effort pour les intégrer dans la politique plus générale de l'entreprise tout en tenant compte des besoins exprimés par les salariés (cela ressort nettement lors des entrevues). La volonté apparente de considérer cette formation à plus long terme permet de rejeter l'idée d'une application ponctuelle de la formation venant résoudre des problèmes au coup par coup.

\subsubsection{Des formations variées}

De manière générale, les PME se heurtent à une législation souvent mal adaptée en matière de formation professionnelle. En effet, «pour qu'une action de formation entre dans le champ de l'obligation légale, il faut qu'elle soit conforme aux caractéristiques de la formation formalisée retenue par la loi » (Verdier, 1991). Or, s'interroger sur la nature des formations suivies revient indirectement à vérifier si les formations entrent dans ce cadre formel. D'après Bel et Rosanvallon (1991), lorsque l'effet de formation augmente, la tendance est plutôt à l'externalisation des stages de formation. De plus, les responsables des entreprises les plus innovantes en formation tendent à combiner les différents types de formation. En effet, l'observation des PME mondiales est très significative d'une approche variée, riche, combinant plusieurs types de formation.

TABlEau 5

Nature des formations

Nature des formations suivies

(plusieurs réponses possibles)

Nombre de

réponses

(\%)

- stages extérieurs de courte durée (moins de 10 jours)

$29(100 \%)$

- formation interne avec formateur externe

$20(69 \%)$

- formation interne avec formateur interne

$17(58 \%)$

- congrès, colloques, etc.

$15(51 \%)$

- stages extérieurs de longue durée

$4(13 \%)$

La combinaison des différents types de formation constatée, concrétisée par un croisement de formations formelles et informelles, peut résulter de l'existence de facteurs particuliers. En effet, pour les PME en permanente innovation sur des créneaux pointus, comme la plupart des PME mondiales étudiées, il semble que certaines formations ne puissent se faire qu'en interne, 
les connaissances étant le fruit de la recherche de l'entreprise elle-même. C'est la raison pour laquelle $86 \%$ des PME font de la formation interne en ayant recours à un formateur interne ou externe.

\subsubsection{Une délégation peu fréquente}

Le problème, présenté plus haut, de la responsabilité de la formation apparaît directement en liaison avec la délégation à des Fonds d'assurance formation $(\mathrm{FAF})^{2}$. Comparativement à d'autres études (AGEFOS, 1992 ; Paradas, 1993), les PME mondiales, globalement, délèguent peu la gestion de leurs fonds destinés à la formation. Dans l'étude de l'AGEFOS (1992), $79 \%$ des PME étudiées adhèrent à un FAF tandis que seulement $51 \%$ des PME de classe mondiale délèguent la formation à de tels organismes. Dans l'étude de Paradas (1993), la délégation par les PME à un FAF est plus souvent en liaison avec les attitudes les plus ponctuelles et les moins intégrées à la stratégie générale. Dans ces conditions, le comportement des PME de classe mondiale autorise à penser que les politiques de formation s'inscrivent dans une logique d'investissement stratégique.

\subsubsection{Un accès à la formation démocratique}

En ce qui concerne l'accès à la formation par les différents niveaux hiérarchiques, tout d'abord, les recherches antérieures semblent souligner que très peu d'ouvriers non qualifiés sont formés dans les PME et que l'inégalité est flagrante en comparaison avec les plus grandes entreprises (Berton et Podevin, 1991 ; Bouayed, 1991). Pour l'AGEFOS (1992), seulement $7 \%$ des salariés seraient formés dans les entreprises de 10 à 19 salariés. La priorité serait laissée aux cadres, puis aux employés qualifiés.

Les résultats obtenus dans la présente enquête (tableau 6) situent les salariés à la première place des personnes formées, suivis de très près par les cadres. La forte dissonance entre ces résultats et les données collectées ailleurs peut s'expliquer par le fait que les PME interrogées ne doivent pas être composées par de nombreux ouvriers non qualifiés, compte tenu des exigences technologiques et des besoins de polyvalence des membres de ces organisations. Thouverez (1990) relève que les PME à haute technologie exportatrices emploient plus de $50 \%$ de cadres que la moyenne des PME : ingénieurs, techniciens supérieurs et cadres commerciaux s'y retrouvent en plus grand

2. Les FAF, en France, ont pour mission de collecter les pourcentages versés au titre de la formation continue, de mutualiser ces sommes et de gérer la mise en œuvre financière des formations dans les entreprises. L'adhésion à ces fonds par les entreprises n'est pas obligatoire. 
nombre. Enfin, ce sont les dirigeants qui se forment le moins, ce qui confirme le constat de Bouayed (1991). Toutefois, selon l'étude de ce dernier, les deux tiers des dirigeants n'ont pas suivi de stage durant les douze derniers mois. Ce point est en forte contradiction avec la déclaration de $79 \%$ des dirigeants des PME de classe mondiale qui affirment suivre des formations.

TABLEAU 6

Catégories formées et priorité de formation

\begin{tabular}{lcc}
\hline $\begin{array}{l}\text { Catégories formées } \\
\text { dans l'entreprise }\end{array}$ & $\begin{array}{c}\text { Nombre d'entreprises } \\
(\%) \\
\text { (plusieurs réponses } \\
\text { possibles) }\end{array}$ & $\begin{array}{c}\text { Catégorie formée } \\
\text { en priorité } \\
\text { dans l'entreprise } \\
\text { (une seule réponse) }\end{array}$ \\
\hline du point de vue hiérarchique : & $29(100 \%)$ & \\
- les salariés & $25(86 \%)$ & $16(55 \%)$ \\
- les cadres & $23(79 \%)$ & $10(35 \%)$ \\
- la dirigeant / direction & & $3(10 \%)$ \\
du point de vue fonctionnel: & $28(96 \%)$ & $13(45 \%)$ \\
- les techniciens & $23(79 \%)$ & $7(24 \%)$ \\
- les chercheurs & $25(86 \%)$ & $5(17 \%)$ \\
- les administratifs & $20(69 \%)$ & $4(14 \%)$ \\
- les commerciaux & & \\
\hline
\end{tabular}

En ce qui concerne les différentes fonctions, les responsables des PME de l'échantillon placent la formation des techniciens en premier lieu suivi des chercheurs, puis des administratifs et enfin des commerciaux. La place de la technique dans les PME étudiées apparaît à nouveau évidente. Finalement, il semble que toutes les catégories de l'entreprise, tant sur le plan hiérarchique que fonctionnel, soient associées à la formation dans les PME mondiales. La multiplication des acteurs en formation est un signe du développement de cette action vers un axe plus innovant (Bel et Rosanvallon, 1991).

\subsubsection{Une perception limitée des obstacles à la formation}

La perception des obstacles liés à la mise en œuvre de la formation donne des indications plus qualitatives sur les modalités des formations engagées. Souvent, les réponses aux questions évoquant les difficultés pour développer la formation ressemblent à une liste de doléances (Garand, 1993, p. 227 ; Fabi et Garand, 1994, p. 338). Or, $38 \%$ des responsables de PME interrogés ne perçoivent pas d'obstacle à la formation. Les obstacles les plus cités sont liés à l'organisation du travail et aux moyens engagés, soit le temps et l'argent. Viennent ensuite les critiques envers les capacités ou la motivation du 
personnel, enfin, les défauts de l'offre de formation et les problèmes liés à l'environnement. Toutefois, $80 \%$ des responsables interrogés se sont montrés satisfaits de l'adaptation des formations proposées par l'offre. Ce résultat reste compréhensible : les formations suivies à l'extérieur sont souvent assez classiques et correspondent à des domaines bien maîtrisés par les organismes de formation. Seules certaines demandes touchent à des domaines technologiques de haut niveau.

Ainsi, les responsables des PME mondiales ont conscience des limites de la formation au sein même de l'organisation et évitent d'en faire rejaillir la responsabilité sur leurs employés ou sur l'extérieur. Cela diffère des résultats obtenus dans la recherche de l'AGEFOS (1992) évoquant des difficultés plus souvent liées au personnel et beaucoup moins à l'organisation ou aux moyens. Enfin, les problèmes relatifs aux difficultés de promotion (Bel et Rosanvallon, 1991) ou à la peur du départ du salarié ne sont jamais cités.

\subsection{Des $P M E$ investissant réellement en formation}

Finalement, les responsables de formation des PME mondiales montrent une maturité certaine dans leur réflexion sur la formation professionnelle. Conscientes de l'impossibilité de faire appel à l'extérieur pour des domaines très pointus, elles intègrent des systèmes de formation interne élaborés, à l'aide quelquefois d'une intégration dans certains réseaux. À côté des connaissances spécifiques, elles utilisent sans trop de difficultés ce qui leur est offert dans des domaines plus classiques.

En nous référant au concept d'investissement formation tel que nous l'avons défini, l'analyse des données laisserait donc entrevoir une existence plus fréquente de cette démarche intégrée, stratégique, réfléchie, dans les PME mondiales. Non seulement les PME observées développent de nombreuses actions de formation, mais elles le font plus dans une perspective d'investissement que dans une logique ponctuelle, ce qui ne semble pas exister dans la majorité des PME françaises.

La question qui se pose alors est: pourquoi ? Quelles caractéristiques des PME étudiées font que la formation est finalement plus et «mieux » développée que dans la majorité des autres entreprises ? L'étude descriptive des variables qui caractérisent les PME mondiales doit s'accompagner d'une réflexion et d'une discussion permettant d'éclaircir les interrogations et de répondre à cette question. 


\section{Caractéristiques des PME mondiales et conséquences sur la formation}

Caron (1994) affirme que, de l'avis des dirigeants québécois, la formation constitue un important facteur de performance de l'entreprise. D'après Doray et al. (1994),

[la formation] possède un impact sur le développement de l'entreprise si elle est articulée aux stratégies de la firme en matière de marché, au développement technologique, au processus de travail ou à la politique d'emploi. En somme, la mise en relation de la formation avec les autres dimensions constitutives de la firme est analytiquement nécessaire.

Aussi, avant de chercher à mieux comprendre les caractéristiques déterminantes de la formation dans les PME mondiales observées, il paraît intéressant de tenir compte des perceptions qu'ont les responsables interrogés de la formation et des relations qu'elle entretient avec les performances de leur entreprise. Le catalogue édité par le club des numéros 1 mondiaux offre des données relatives aux raisons perçues par les dirigeants de la performance de leur entreprise et de leur position de leader ; l'exploitation de ces informations enrichit l'analyse de l'échantillon.

\subsection{Des motivations liées à l'activité de l'entreprise}

Il apparaît principalement que le profil d'activité de l'entreprise incite les responsables à mener les formations décrites. En effet, dans un environnement turbulent où les technologies, les marchés, la législation et les besoins des clients évoluent en permanence, dans lequel l'adaptation et la flexibilité sont les clés de la réussite, la formation semble avoir une place privilégiée. Ainsi, les responsables affirment avoir un besoin de formation accru pour conserver leur place de leader sur le marché (83\%), pour s'adapter aux clients et aux normes $(79 \%)$ et pour répondre à une innovation permanente $(76 \%)$. Les raisons liées à l'organisation générale sont beaucoup moins souvent citées comme catalyseur de formation. Ainsi, le caractère international des transactions ou la recherche de polyvalence n'apparaissent pas pour les responsables comme des problèmes pouvant être résolus par la formation.

D'après les données du tableau 7 , ce n'est pas directement le caractère mondial qui accroît les besoins d'investissement en formation, mais plutôt les exigences de cette position mondiale, par exemple, la résistance à la turbulence de l'environnement, la recherche permanente d'une position de leader et la volonté d'exécuter parfaitement sa mission. "Le juge ultime de la compétitivité industrielle est donc le marché. » (Forum économique mondial, 1985) Toutefois, ces premières analyses de la perception des besoins par les responsables des 
TABLEAU 7

Raisons de la formation

Raisons incitant l'entreprise à faire de la formation (plusieurs réponses possibles)

Nombre

de réponses

(\%)

$24(83 \%)$

$23(79 \%)$

$22(76 \%)$

$18(62 \%)$

$17(59 \%)$

$16(55 \%)$

PME mondiales ne semblent pas suffisantes pour tirer des conclusions sur les caractéristiques déterminantes de l'investissement en formation. Une analyse plus fine des caractéristiques de ces PME demeure nécessaire.

\subsection{Des pratiques particulièrement innovantes}

Pour les deux tiers des PME de l'échantillon, l'innovation permanente, le dépôt de un ou de plusieurs brevets et une forte recherche et développement constituent une clé de leur succès. Même si l'innovation ne détermine pas à elle seule la performance de l'entreprise, elle constitue la raison la plus souvent avancée comme facteur de réussite par les dirigeants $(65 \%)$. Cette forte capacité des PME mondiales à innover s'explique en partie par le profil des dirigeants des PME étudiées : $75 \%$ des dirigeants sont soit des ingénieurs ou des détenteurs de doctorat dans des domaines techniques ou scientifiques, soit des détenteurs de diplômes universitaires dans des domaines économiques et commerciaux. Il convient toutefois de préciser que $20 \%$ des PME sont dirigées par des autodidactes, sans que cela puisse avoir une influence sur la capacité d'innovation de l'entreprise. Ainsi, même si l'éducation du dirigeant peut créer une prédisposition favorable de l'entreprise à innover, elle n'en constitue pas la seule explication.

De manière assez générale, l'innovation engendre un besoin d'acquisition et de renouvellement des savoirs et des connaissances dans l'entreprise, aussi bien au stade de l'invention qu'à celui de la diffusion (Nooteboom, 1994). La formation peut évidemment contribuer à satisfaire ces exigences. L'importance accordée à l'innovation par le dirigeant influence largement les actions de formation, au-delà du simple caractère mondial de l'entreprise. À ce sujet, Bayad (1993) affirme que «le pouvoir discriminant de la dimension humaine et de l'orientation client est à la base des potentialités internes et externes de la PME en matière d'innovation. L'implication qui en découle est aussi une stratégie de formation et d'implication de l'homme dans l'entreprise. » 
L'innovation apparaîtrait davantage comme un facteur explicatif de la politique de formation de l'entreprise.

\subsection{Des PME souvent très spécialisées}

Pour $40 \%$ des responsables interrogés, la performance de leur PME tient à une forte spécialisation. "L'hyper-spécialisation dans des créneaux étroits peut être considérée comme une des composantes de la réussite des PME, dans la mesure où elle limite les conflits concurrentiels et assure aux firmes le leadership de leur spécialité. » (Mérigot et Gautreau, 1986) Nous voyons apparaître une situation de quasi-monopole, ce que Penrose qualifie de «monopole interstitiel» (Marchesnay, 1990). À son tour, Saporta (1986) a insisté sur la séquence spécialisation-internationalisation des PME, surtout lorsque le marché national est insuffisant pour permettre à l'entreprise d'atteindre son seuil de rentabilité (80\% des PME mondiales observées se trouvent dans cette situation). Dans ces conditions, l'engagement international traduit davantage une nécessité qu'une option stratégique.

La forte spécialisation des PME de l'échantillon ressort plus clairement à l'observation des activités d'importation, développées par près de $60 \%$ des entreprises. Parmi les raisons invoquées, une très grande proportion des dirigeants $(85 \%)$ éprouvent le besoin d'importer, car les produits, machines ou composants nécessaires à l'activité n'existent pas en France.

Ce fort degré de spécialisation influence les politiques de formation des PME mondiales. Le problème de variété des types de formation et de difficulté des choix de formation trop spécifiques à l'extérieur a déjà été évoqué (Garand et Fabi, 1992) : $86 \%$ des entreprises étudiées font de la formation interne avec formateur interne ou externe. Ainsi, dans de nombreux cas, la formation ne peut se faire à l'extérieur du cadre de l'organisation.

\subsection{Une compétitivité fondée sur la technologie}

La technologie est considérée comme un facteur de compétitivité internationale par $55 \%$ des entreprises étudiées, ce qui semble logique dans un contexte où de plus en plus de technologies se créent et se diffusent à l'échelle mondiale (Fourcade, 1993), entretenant l'expression de «technoglobalisme » développée par les experts de l'OCDE. Pour les PME, «la compétitivité passe de plus en plus par la maitrise de la technologie, que celle-ci soit liée aux produits, aux processus ou à la gestion de toute la production [...] or, la diffusion des nouvelles technologies peut être facilitée par la présence d'un système d'enseignement ou de formation générale ou continue particulièrement orientée vers le changement» (Jacob et Julien, 1993). Ainsi, «la formation en 
entreprise devient un outil stratégique d'adaptation du personnel aux nouvelles technologies » (Doray et al., 1994). Les recherches de Caron (1994) accentuent ces affirmations, l'auteur établissant un lien entre formation et développement technologique. Il affirme que le recours de plus en plus fréquent aux technologies nouvelles constitue un atout exceptionnel dans un nouveau contexte où la compétition se joue désormais sur un terrain international. «C'est en étroite articulation à ces objectifs que la formation continue prend sa pleine valeur comme élément de changement dans l'entreprise.» Dans ce contexte, les besoins de formation s'accroissent, ce que confirment les deux tiers des dirigeants de l'échantillon, pour lesquels l'aspect technologique constitue une raison principale qui les pousse à faire de la formation.

En outre, veille technologique, flexibilité technique et maîtrise d'un savoir-faire technique apparaissent comme des éléments caractéristiques des PME observées. L'acquisition d'avantages technologiques est liée à l'intensité de la R-D, orientée dans la production de biens à forte valeur ajoutée (Forum économique mondial, 1985). Si la technologie reste un facteur important de réussite, elle « ne suffit pas à améliorer la compétitivité des PME. Il faut que cette introduction soit associée à l'innovation de produits et à la formation des ressources humaines, ou à divers autres éléments [...] d'investissement immatériel » (Jacob et Julien, 1993).

\section{Synthèse et conclusion}

\subsection{Des PME mondiales innovantes et dynamiques}

Il apparaît clairement que les PME étudiées se situent dans le groupe des PME dites «innovantes en formation» (Bel et Rosanvallon, 1991). En effet, elles présentent comme caractéristiques principales de formation une réflexion dans la durée, une formalisation poussée, une multiplication des objectifs, des formes et des acteurs de formation. En un sens, on retrouve la « combinaison pragmatique » mise en évidence par Sarnin (1990) à propos des pratiques de formation occasionnées par des changements stratégiques, au rang desquels figure l'internationalisation.

Toutefois, les responsables n'ont pas invoqué comme raison première de ces politiques de formation l'internationalisation de leurs activités. Par contre, les exigences liées au marché et à ses caractéristiques, telles que la technologie, l'innovation et la spécialisation, semblent perçues à la fois comme des facteurs de performance et des vecteurs de formation. Elles constituent en même temps des causes et des conséquences communes à la formation et à la mondialisation. La technologie, l'innovation et la spécialisation, indissociables du caractère mondial des PME étudiées, impliquent des besoins de formation plus 
importants et spécifiques, et donc une politique de formation ayant un caractère d'investissement marqué. La compétitivité mondiale reste du ressort de deux catégories de facteurs : ceux qui échappent au contrôle direct des entrepreneurs (comme les ressources naturelles ou l'influence de l'État) et ceux que les hommes d'affaires peuvent influencer, dont l'éducation et la formation, qui présentent un rapport à plus long terme (Forum économique mondial, 1985). Cela confirme la logique d'une approche d'investissement permettant une rentabilité à long terme.

\subsection{La formation adaptée aux exigences des marchés}

Les PME observées se démarquent principalement par la diversité de leurs politiques de formation. En effet, elles semblent relier sans difficulté des aspects de formation formels et informels, et cela par nécessité. Ces résultats rejoignent l'analyse de Marchesnay (1993) selon laquelle:

[...] la globalisation va entraîner une grande spécificité du couple métiermission pour les petites entreprises. Ainsi, leurs bases de compétitivité reposent sur une forte idiosyncrasie dans l'échange. Cela implique que la petite entreprise va privilégier un ensemble de tâches au sein de l'organisation, s'y consacrer exclusivement, quitte à externaliser les autres tâches, lesquelles risquent d'être mieux assumées par d'autres.

En d'autres termes, les PME de l'échantillon externalisent une partie de la formation avec moins de réticence que la majorité des PME. Ces formations effectuées en dehors de l'entreprise s'inscrivent dans le cadre formel de l'obligation légale. En revanche, toute une série de formations ne peuvent être assurées qu'à l'intérieur de l'entreprise, pour des raisons déjà évoquées de spécialisation accrue et d'innovation permanente. C'est ainsi que les cadres, le chef d'entreprise, ou des chercheurs se retrouvent formateurs, les dépenses engendrées par ces formations n'étant généralement pas prises en compte dans le cadre de l'obligation. Tantôt défensive, par sa spécialisation et son maintien dans des niches, tantôt prospective, par la nouveauté de son produit ou service (Rousseau et Wade Benzoni, 1994), la PME de classe mondiale développera une politique de formation externe extensive (maîtriser son métier) couplée à une formation «sur le tas » importante, liée à l'innovation.

Finalement, la loi française semble mal adaptée à certaines situations de formation. Bien que conçue pour développer la formation dans toutes les structures et particulièrement dans la PME, la législation ne favorise pas d'autres formes de formation que celles adaptées plus fondamentalement à la grande entreprise (Garand, 1993, p. 229). L'apparition de l'obligation pour les PME de moins de 10 salariés relance de façon encore plus forte ce débat et pose le problème de l'efficacité de ce type de mesure, sans que soient prises en considération les véritables spécificités des plus petites entreprises. 


\subsection{Limites et pistes de recherche}

\subsubsection{Limites d'ordre méthodologique}

Un premier problème de méthode réfère au nombre réduit de recherches dans le domaine. D'une part, les enquêtes servant de base de comparaison ont un caractère hétérogène, ce qui a limité les possibilités d'analyse. De plus, la comparaison reste nationale. La législation française en matière de formation est particulière, mais l'étude d'autres travaux d'origines différentes pourrait permettre la mise en évidence ou la confirmation des déterminants de la politique de formation en PME. En outre, l'analyse présentée reste synchronique, statique. Or, une telle étude aurait peut-être mérité une approche diachronique, considérant plutôt l'ensemble des faits du point de vue de leur évolution. Les pratiques de formation s'inscrivant dans le temps, la prise en compte de cette dimension peut s'avérer un prolongement fécond des résultats obtenus dans cette étude.

Enfin, les résultats obtenus s'appuient sur un échantillon très spécifique de PME, ce qui semble justifié par l'objet même de notre recherche, mais impose de rester très prudent quant aux possibilités d'extrapolation. Par exemple, Sarnin (1990), dans une étude concernant les relations entre changements stratégiques et pratiques de formation, avance des conclusions qui doivent inciter à la prudence : «il est plus délicat d'évaluer les résultats de l'effort de formation sur le plan d'une stratégie tournée vers l'internationalisation. Les cas étudiés indiquent que c'est dans ce dernier domaine que les politiques de formation sont les plus réduites. » Les contradictions apparentes devront se comprendre à travers la multiplication des recherches dans le domaine, mais aussi grâce à la précision de certains concepts nouveaux, comme celui de globalisation, qui mérite une opérationnalisation plus détaillée.

\subsubsection{Analyse des variables exogènes}

Dans la présente étude, les variables concernent tout particulièrement le caractère mondial des PME. Toutefois, il ne semble pas possible de se limiter à ces seules données, dès lors que l'on désire évaluer des facteurs d'influence sur les modalités de formation. D'autres variables, qualifiées ici d'exogènes, relativement au statut qui leur est donné, peuvent intervenir dans la politique de mise en œuvre des actions de formation professionnelle.

Une première variable exogène à considérer concerne le profil du dirigeant et plus particulièrement sa formation. Plus celui-ci aura un niveau de formation élevé, plus il sera sensibilisé à de nouvelles formations, pour lui ou pour ses salariés (Kalifa et Paradas, 1994). Or, $75 \%$ des dirigeants déclarent posséder un diplôme universitaire de cycles supérieurs. Le niveau élevé de 
formation initiale des dirigeants constitue une prédisposition favorable à la mise en place de formations professionnelles. De plus, certaines tendances non appréhendées dans le questionnaire, telles que la vision du dirigeant ou son histoire, peuvent également constituer des limites dans la précision de l'enquête.

La seconde variable porte sur l'activité des entreprises, car la plupart des PME mondiales étudiées ( $88 \%$ ) sont des PME industrielles qui développent des activités de fabrication et tendent à avoir plus souvent que les autres un plan de formation. D'autres conséquences non identifiées peuvent intervenir dans ce domaine, en termes de formation, et doivent inciter à la prudence quant aux conclusions obtenues.

Si la taille ne constitue pas, dans le cadre de la présente étude, une variable déterminante de l'ensemble de la politique de formation, elle n'en reste pas moins influente sur certains points. La formalisation du plan, par exemple, existe dans $36 \%$ des PME de moins de 50 salariés et dans $93 \%$ des PME de plus de 50 salariés de l'échantillon étudié. Il est toutefois nécessaire de tenir compte des obligations liées à la formation et au rôle du comité d'entreprise (à partir de 50 salariés) dans ce processus de formalisation. Il n'en reste pas moins que les petites entreprises se caractérisent par un système de gestion peu structuré et peu formalisé (Julien et Marchesnay, 1988). Bien que la formalisation ne constitue pas le seul indicateur d'une politique d'investissement en formation, les résultats de l'enquête laissent entrevoir l'existence d'une relation entre la taille et la formalisation de la formation. Enfin, la maturité peut également exercer une influence sur les politiques de formation. Des croisements avec l'âge des PME étudiées montrent que cette variable n'apparaît pas du tout comme déterminante dans l'échantillon observé: le pourcentage de la masse salariale et l'existence d'un plan ne dépendent pas, dans la présente recherche, de la maturité de l'entreprise.

D'autres variables pourraient aussi intervenir dans le processus de détermination de la politique de formation, sans que nous n'ayons pu les identifier. Il convient de citer, par exemple, les pratiques d'embauche et de recrutement qui peuvent avoir une forte influence sur le niveau des compétences au sein de l'entreprise (Bayad et Paradas, 1995), et donc sur la politique de formation. Ainsi, Bayad et Herrmann (1991) signalent un lien entre politique favorable à l'embauche et absence de formation professionnelle particulière. De plus, les pratiques d'embauche apparaissent elles-mêmes en très forte relation avec la technologie et en relation sensible avec l'innovation. Une autre étude, engagée au Royaume-Uni, explique que « les marchés de la main-d'œuvre fonctionnent de façon à réduire la nécessité et l'efficacité des programmes de formation dans les entreprises plus prospères. Ces dernières sont plus susceptibles que les entreprises moins prospères d'engager des personnes de l'extérieur. Les premières ont tendance à accroître le niveau de compétence par le recrutement 
de personnes qualifiées de l'extérieur, tandis que les entreprises du groupe apparié sont plus susceptibles de développer les compétences internes » (Baldwin, 1994). Ce constat impose une limite évidente à toute recherche engagée dans des domaines complexes et encore peu explorés, où une multitude de variables interviennent. Des études ultérieures devront donc répondre à ces interrogations en précisant l'importance relative de nouvelles variables dans la mise en œuvre de politiques de formation.

\section{Bibliographie}

AGEFOS-PME (1992a), «Perspectives 93. Les PME face aux ressources humaines et à la formation », Sondage national auprès de 450 entreprises, novembre, Document AGEFOS-PME, $52 \mathrm{p}$.

AGEFOS-PME (1992b), «Besoins des PME en conseil pour la GRH et la formation », Conférence nationale, novembre, $88 \mathrm{p}$.

BALDWIN, J. (1994), « Stratégies de réussite, profil des PME en croissance au Canada », Statistiques Canada, Ministère de l'Industrie, du Commerce, des Sciences et de la Technologie, $110 \mathrm{p}$.

BAYAD, M. (1993), «L'effort de recherche de la PME industrielle face au développement des produits nouveaux : la prépondérance des moyens humains ", Revue Internationale PME, vol. $6, \mathrm{n}^{0} 1$, p. 29-48.

BAYAD, M. (1992), «Internationalisation de la PME et pratiques de gestion des ressources humaines ", Actes du troisième Congrès de l'AGRH, novembre, Lille, p. 390-398.

BAYAD, M. et J.L. HeRrmanN (1991), «Gestion des effectifs et caractéristiques des PME industrielles: vers quelles relations?», Revue Internationale PME, vol. $4, \mathrm{n}^{0} 2$, p. 5-41.

BAYAD, M. et A. PARADAS (1995), «Les difficultés de recrutement en PME: recherche sur les déterminants organisationnels », dans D'Arcimoles et al. (éd.), Transversalité de la GRH, Actes du sixième Congrès de l'AGRH, Poitiers, France, 23-24 novembre, p. 668-679.

BecCarelli, C. et H. PerKer (1991), «PME-PMI : définitions et impact socioéconomique ", Actualité de la Formation Permanente, no 114, p. 36-37.

Bel, M. et A. Rosanvallon (1991), «Les politiques innovantes : quelles dynamiques?», Actualité de la Formation Permanente, $\mathrm{n}^{0}$ 114, p. 53-57.

BeL, M. et A. Rosanvallon (1990), «La politique de formation dans les PMEPMI », Document IREPD, Université des sciences sociales de Grenoble, $130 \mathrm{p}$.

BerRa, L., PiatTI, L. et G. Vitali (1995), «The internationalization process in the small and medium sized firms : a case study on the Italian clothing industry », Small Business Economics, vol. 7, p. 67-75. 
Berton, F. et G. Podevin (1991), «Vingt ans de formation continue: de la promotion sociale à la gestion de l'emploi », Formation Emploi, n ${ }^{0} 34$, p. 14-30.

BouAyed, A. (1991), « Agefos-PME: deux années de sondage-formation des entreprises », Actualité de la Formation Permanente, no 114, p. 38-41.

Bucaille, A. et B. Costa DE BEAUREgARd (1987), PMI, enjeux régionaux et internationaux, Paris, Economica, 327 p.

CARON, J. (1994), «Formation continue et entreprise dans le nouveau contexte économique », Québec, Secrétariat du Conseil de la science et de la technologie, novembre, $49 \mathrm{p}$.

CASPAR, P. et C. AfRIAT (1988), L'investissement intellectuel, Paris, Economica, p. $40-50$.

CASTAGnos, J.C. (1990), « Technologie et formation continue : l'amorce d'une relation stratégique », Cahiers de recherche du CERAG, no 90-10, 24 p.

CAVUSGIL, T.S. (1984), "Organizational characteristics associated with export activity », Journal of Management Studies, vol. 21, n 1, p. 3-22.

CEREQ BREF (1992), «Bulletin de recherche sur l'emploi et le formation », n 77, juin.

D’AMBoise, G. (1994), «La mise en œuvre des plans et des stratégies », Document de travail, 94-19, Université Laval, 26 p.

D'Amboise, G. et BAKAniBona (1990), «La planification dans les PME», Revue Internationale $P M E$, vol. $3, \mathrm{n}^{0} 2$, p. 148-165.

D'AmBoise, G. et C. FoRTIN (1990), «Proposition d'une grille de classification pour l'identification de la logique stratégique des petites et moyennes entreprises », Document de travail, 90-15, Université Laval, 32 p.

DeJeAn, P. et J.P. Willems (1991), « De la gestion des sommes à la gestion des hommes », Actualité de la Formation Permanente, $\mathrm{n}^{0} 114$, p. 69-72.

DERoY, X. et L. LANGRAND-EsCuRE (1992), «Essai de précision du concept de globalisation: application au secteur de la télévision haute-définition », Document de recherche, École supérieure de commerce de Reims, France, 29 p.

Doray, P., BAGAOUY, R. et D. RICARD (1994), «La formation dans les entreprises québécoises : études de cas auprès de 15 entreprises performantes », Rapport soumis au Conseil de la science et de la technologie du Québec, novembre, $195 \mathrm{p}$.

FABI, B. et D.J. GARAND (1994), «La gestion des ressources humaines dans les PME », dans Julien et al., Les PME : bilan et perspectives, Québec, Les Presses Inter Universitaire; Paris, Economica, p. 297-368.

FORUM ÉCONOMIQUE MONDIAL (1985), Rapport sur la compétitivité internationale, Paris, Economica, p. 6-7. 
FOURCADE, C. (1993), «Petites entreprises innovantes et réseaux transnationaux », Cahiers de l'ERFI, $\mathrm{n}^{0}$ 1, Montpellier, p. 25-44.

FouRCADE, C. (1986), «Le démarrage des entreprises: une comparaison internationale», dans P. A. Julien, P. Chicha, et A. Joyal, Les PME dans un monde en mutation, Sainte-Foy, Presses de l'Université du Québec, p. 253-277.

Fuguet, J.L., Peguin, D., Renard, M.F. et N. RiChez-BAtTesti (1986), «L'impact de la contrainte extérieure sur des zones d'activité urbaines ou locales », Aixen-Provence, Rapport final pour la DATAR, CEFI, 218 p.

GARAND, D.J. (1993), Les pratiques de gestion des ressources humaines (GRH) en petites et moyennes entreprises (PME) : une synthèse conceptuelle et empirique, Rapport de recherche, Groupe de recherche en économie et gestion des PME (GREPME), Département d'administration et d'économique, Université du Québec à Trois-Rivières, $386 \mathrm{p}$.

GARAND, D.J. et B. FABI (1992), «État de la recherche. Les pratiques de gestion des ressources humaines (GRH) en PME », Revue Organisation, vol. 2, nº 1, p. 61-99.

Guilhon, A., Guilhon, B. et D. Peguin (1993), «L'identité de la PME à travers l'activité d'exportation ", Actes du Congrès international francophone de la $P M E$, Carthage, Tunisie, 28-30 octobre, p. 301-312.

HERMEL, P. (1991), «Développement international et management des ressources humaines », Actes du deuxième Congrès de l'AGRH, Cergy, p. 373-381.

JACOB, R. et P.A. JULIEN (1993), «Les nouvelles technologies », dans Julien et al., Les PME : bilan et perspectives, Québec, Les Presses Inter Universitaire ; Paris, Economica, p. 189-224.

JOFFRE, P. (1994), Comprendre la mondialisation de l'entreprise, Paris, Economica, Collection Poche, 155 p.

JOFFRE, P. (1987), L'entreprise et l'exportation, Paris, Éditions Vuibert, 144 p.

JoYAL, A. et C. RAMANGALAHY (1993), « Typologie des comportements stratégiques des PME exportatrices: une étude de cas », Actes du Congrès international francophone de la PME, Carthage, Tunisie, 28-30 octobre, p. 313-324.

Julien, P A. et M. MARCheSNAY (1988), La petite entreprise, Paris, Éditions Vuibert, $288 \mathrm{p}$.

KAlifa, M. et A. PARADAS (1994), «La recherche d'un contexte propice à la mise en place de formations professionnelles dans les TPE», Actes du cinquième Congrès de l'AGRH, novembre, Montpellier, p. 611.

LEVITT, T. (1983), «Un seul univers : le marché ? », Harvard l'Expansion, automne, p. 6-17.

MAthe, J.C. et A. Rivet (1988), «Investissement stratégique dans les moyennes entreprises ", Revue Internationale PME, vol. 1, n ${ }^{0} 4$, p. 309-325. 
MALIVERNAY, J. (1991), «Les entreprises exportatrices : une concentration accrue », Économie et statistique, $\mathrm{n}^{0} 244$, juin, p. 15-24.

MARCHESNAY, M. (1990), «Les stratégies de spécialisation », Encyclopédie du Management, Tome 2, Paris, Éditions Vuibert, p. 773-780.

MARCHESNAY, M. (1993), «Les bases de compétitivité de la petite entreprise face à la globalisation des marchés », Cahiers de l'ERFI, no 1, Montpellier, p. 9-24.

Merigot, J.C. et M.C. Gautreau (1986), « Oscars de l'exportation: les lauréats restent compétitifs », Revue Française de Gestion, n 55, p. 106-117.

MESCHI, P.X. (1993), «Pratique de formation dans les entreprises françaises : caractéristiques et contingence organisationnelle », Actes du quatrième colloque de l'AGRH, Jouy-en-Josas, p. 333-343.

MoINI, A.H. (1992), «Europe 1992 : a challenge to small exporters », Journal of Small Business Management, vol. 30, $\mathrm{n}^{0}$ 1, p. 11-20.

MONNOYER-LONGÉ, M.C. (1990), «L'organisation, clé de la performance », dans P.Y. Léo, M.C. Monnoyer-Longé, et J. Philippe, Stratégies internationales des $P M E$, Paris, Economica, 263 p.

Nooteвоom, B. (1994), «Innovation and diffusion in small firms: theory and evidence », Small Business Economics, vol. 6, nº 5, octobre, p. 339-342.

PARADAS, A. (1993), «Contribution à l'évaluation de la formation professionnelle dans les PME », Thèse de doctorat, Université de Montpellier I, 495 p.

PORTER, M.E. (1993), L'avantage concurrentiel des nations, Paris, Interéditions, 883 p.

REICH, R. (1993), L'économie mondialisée, Paris, Dunod, 310 p.

Rousseau, M.D. et K.A. WADE-BENZONI (1994), «Linking strategy and human ressource practices : how employee and customer contracts are created », Human Resource Management, vol. 33, n 3, p. 464.

SAPORTA, B. (1993), «Les enjeux de l'ouverture internationale des PME ». Communication au colloque «PME-PMI, développement international », 9-11 juin, Aix-en-Provence, p. 811-825.

SAPORTA, B. (1986), «Les PME-PMI face au développement international », Cahiers de recherche de l'IAE de Toulouse, $\mathrm{n}^{\circ}$ 50, mai, $52 \mathrm{p}$.

SARNIN, P. (1990), «Changements stratégiques et pratiques de formation dans 16 PMI de Rhône-Alpes», IRE, Colloque TETRA «La PME: objet de recherche pertinent ? », 30-31 mai, Lyon, 13 p.

TAChe, A. et P. Dejean (1991), «Actualité des représentations de la formation continue et des outils multimédias : les PME-PMI de la région Midi-Pyrénées », CRIDF, Université des sciences sociales de Toulouse, $136 \mathrm{p}$. 
ThouvereZ, M. (1990), « Haute technologie et exportation, synergies et contraintes d'une double stratégie », dans P.Y. Léo, M.C. Monnoyer-Longé, et J. Philippe, Stratégies internationales des PME, Paris, Economica, $263 \mathrm{p}$.

TORRÈs, O. (1994), « Stratégie de globalisation des petites entreprises et territoire », Cahiers de l'ERFI, $\mathrm{n}^{\circ}$ 7, Montpellier, septembre, p. 20-38.

VENDEUIL (de), R. (1992), «À qui profite la formation continue? », l'Express, 17 septembre, p. 4-10

VERDIER, E. (1991), «Les PME acteurs spécifiques de la formation? », Actualité de la Formation Permanente, $\mathrm{n}^{0} 114$, p. 42-47.

VERDIER, E. (1990), «Efficacité de la formation continue dans les PME » Sociologie du Travail, $\mathrm{n}^{0} 3$, p. 295-320.

WAGNER, J. (1995), «Exports, firm size, and firm dynamics», Small Business Economics, vol. 7, p. 29-39.

WALTERS, P. et S. SAMIEE (1990), «A model for assessing performance in small U.S. exporting firms », Entrepreneurship: Theory and Practice, vol. 15, n ${ }^{0} 2$, p. 33-50.

WAlther, R. (1988), «Former pour investir», Éducation Permanente, n 95 , décembre, p. 51-62. 